Article

\title{
The Increasing Prevalence of Gastroschisis: Associated Factors, Possible Mechanisms, and Potential Mitigative Interventions
}

Claude Hughes ${ }^{*}$ and Obinna O. Adibe ${ }^{2}$

1Therapeutic Science and Strategy Unit, IQVIA; Department of Obstetrics and Gynecology, Duke University Medical Center; Epidemiology and Environmental Epigenetics Laboratory, North Carolina State University, Durham, NC, USA

2Departments of Surgery and Pediatrics, Duke University Medical Center, Durham, NC, USA

Received October 23, 2018; Accepted January 28, 2019

\section{ABSTRACT}

Background: Gastroschisis has increased globally over recent decades and this increase is not explained by demographic changes in maternal age. Implicated risk factors for this increase include lifestyle behaviors, environmental exposures, lower socioeconomic status, lower body mass index, poor nutrition, smoking tobacco, using illicit drugs, alcohol, or analgesics and genitourinary infections.

Methods: Selective review of the literature.

Results: Present hypotheses would only suggest avoidance of suspect exposures as protective interventions. To identify safe and efficacious protective therapies, new cellular/molecular modes-of-action need to be considered. Plausible developmental modes-of-action include a) changes in epigenetic programming of relevant stem or progenitor cells; b) mechanical forces (cellular mechanosensitivity and mechanotransduction) signaling; and c) ephrin-Eph receptor multimodal signaling. These developmental modes-of-action present plausible options for "druggable" molecules that could be developed into protective or mitigative therapeutic agents for gastroschisis.

Conclusion: Possible interventions for modifiable factors in gastroschisis include 1) Delay childbearing. 2) Improve nutrition for younger gravidas. 3) Pre-conceptional counseling to reduce embryonic exposures to the range of implicated lifestyle, environmental and medical factors. 4) Urge research colleagues to investigate the cellular and molecular mechanisms underlying gastroschisis and to translate those insights into one or more safe and efficacious preventive or mitigative therapies.

\section{KEYWORDS}

Druggable molecules; ephrin-Eph receptor; exposures; gastroschisis; mechanosensitivity and mechanotransduction; protective therapeutics; risk factors; translational toxicology

\section{OUTLINE}

I. Introduction

1) The alarming demographic trend in gastroschisis

2) Clinical care, surgery and outcomes

3) Developmental factors and genomic modes-of-action (MOA)

4) Genetic, genomic, gene variants, epigenetics MOAs

II. Risk factors

1) Maternal illnesses, medication use and substance abuse

2) Maternal nutrition and metabolic milieu

3) Environmental exposures

4) Distinct exposure category-agricultural chemicals

III. Potential MOA hypotheses

1) MOA-mechanical forces - mechano-sensitivity and mechano-transduction signaling
2) MOA - Ephrin-Eph receptor signaling

IV. Prospects for protective/mitigative therapies: research and development needs

V. Summary

References

\section{INTRODUCTION}

The alarming demographic trend in gastroschisis

Gastroschisis (GS) is a congenital abdominal wall defect in which the intestines, and sometimes, other organs, protrude outside of the fetal abdomen through an opening in the abdominal wall. The prevalence of gastroschisis is on the rise, increasing two to four times in the last 45+ years in several regions around the world. Data spanning the years of 1970-2015 demonstrate some variation ov-

\footnotetext{
*Correspondence: Claude Hughes, Email: claude.hughes@iqvia.com
} 
er time, but in general, this worrisome trend shows a moderate ongoing increase $[1 ; 2 ; 3 ; 4 ; 5 ; 6 ; 7]$.

While the observed increases in gastroschisis prevalence are not explained by demographic changes in maternal age or race/ethnicity, gastroschisis is strongly associated with young maternal age. The association of gastroschisis with young maternal age is most apparent among mothers aged younger than 20 years. Nonetheless, Jones et al. [3] documented significant increases in prevalence in all age groups during 2006-2012 compared with 1995 -2005. These investigators also noted the most significant increases occurred among younger Black mothers even though the prevalence in Black mothers remains lower than in White or Hispanic mothers.

The underlying cause or causes of these increases have not been definitively established. Some proposed risk factors include lifestyle behaviors, environmental exposores, lower socioeconomic status, and lower body mass index, poor nutrition, smoking tobacco, using illicit drugs, alcohol, or analgesics as well as genitourinary infections.

\section{Clinical care, surgery and outcomes}

The outcome for a fetus or infant who has gastroschisis may be a stillbirth, neonatal death or uncomplicated surgical correction. The Centers for Disease Control and Prevention (CDC) estimate is that about 1,900 babies are born each year in the United States with gastroschisis [1]. While 1-year survival rates may be approximately about $70 \%$ in some regions [5], if delivery of the infant occurs at a tertiary care center with immediate neonatal intervention, life expectancy for infants with gastroschisis can exceed $90 \%$. In virtually all cases, care of affected infants exacts a significant emotional toll on parents and other family members and imposes significant financial/health care costs.

Keys et al. [8] performed a retrospective analysis in the United Kingdom of all patients admitted to a tertiary neonatal surgical center with gastroschisis from January 1996 to December 2005. The primary outcome measures were incidence, length of hospital stay, and the total cost for all patients each year.

Over that interval of ten years, the incidence of gastroschisis rose 3 -fold while the median cost per patient remained relatively constant. Due to the increased incidence of this malformation, the estimated annual cost to the National Health Service (NHS) to care for this condition in England and Wales had risen from $£ 3.6$ million in 1996 to more than $£ 15$ million in 2005 .

Gastroschisis is defined as a full-thickness congenital abominal wall defect usually situated on the right side of the umbilicus, with intestines protruding into the amniotic fluid without any protective membrane. The amniotic fluid creates an inflammation of the bowel wall, called perivisceritis. Associated with intestinal abnormalities are malrotation and a degree of short bowel, volvulus, perforation and atresia may be found [9]. The optimal management of neonates with gastroschisis is unclear, and there is significant morbidity [10]. Surgical mana- gement includes techniques for primary repair in which the intestinal contents are immediately reduced into the abdomen, or silo placement and delayed repair if the abdominal cavity is not able to accommodate the volume of the intestine [11].

Infants with gastroschisis often need other complementary treatments including intravenous nutrients, prophylactic antibiotics, and careful control of body temperature. In utero exposure of the fetal intestine to the amniotic fluid may cause inflammation and bowel injury, resulting in significant gastrointestinal problems during the neonatal period after closure of the defect. Complications include prolonged ileus, sepsis, associated intestinal atresia, malabsorption, wound infection, and necrotizing enterocolitis [11].

Kassa and Lilja [10] conducted a single-center retrospective analysis of 79 neonates with gastroschisis spanning 1989-2009. Length of hospital stay (LOS), days of parenteral nutrition (PN), and survival were outcome measures. Overall survival was $92 \%$, and primary closure was achieved in $80 \%$. Median LOS was 25 days, and median duration of PN was 17 days. Intestinal atresia, "vanishing" gastroschisis, delayed repair, and prematurity all affected outcome. Route of delivery and associated malformations were not related to poorer outcome. Necrotizing enterocolitis did not occur in any of these patients. Outcomes were favorable as measured by survival, LOS, and days of PN. Primary predictors of poor outcome were factors related to short bowel syndrome and delayed repair.

Bergholz et al. [12] compared the outcome of newborns with simple (sGS) and complex gastroschisis (cGS: gastroschisis with intestinal atresia, necrosis, perforation, and/or volvulus) by conducting a systematic database search, quality assessment and meta-analysis of relevant articles which evaluated the mortality and morbidity of newborns with cGS versus sGS. Of 19 identified reports, 13 eligible studies were included. The mortality of infants with cGS (16.67\%) was significantly higher than sGS (2.18\%, RR: 5.39). Infants with cGS are started on enteral feedings later and they take longer to full enteral feedings with a subsequent longer duration of parenteral nutrition. Their risk of sepsis, short bowel syndrome and necrotizing enterocolitis is higher. They stay longer in the hospital and are more likely to be sent home with enteral tube feedings and parenteral nutrition.

de Buys Roessingh et al. [9] performed a retrospective study covering the period from January 2000 to December 2007. The following variables were analyzed for each child: weight, sex, APGAR (Appearance, Pulse, Grimace, Activity, and Respiration) score, perforations, atresia, volvulus, bowel length, subjective description of perivisceritis, duration of parenteral nutrition, initiation of enteral nutrition, time to full enteral intake, necrotizing enterocolitis, the average period of hospitalization, and mortality. Sixty-eight cases of gastroschisis were studied that included 9 cases of perforations, 8 of volvulus, 12 of 
atresia and 49 with a subjective description of perivisceritis (72\%).

The mortality rate was $12 \%$ (eight deaths) and the average duration of total parenteral nutrition was $56.7 \mathrm{~d}$ (min -max: 8-950; median: 22), with five cases of necrotizing enterocolitis. The average length of hospitalization for 60 patients was $54.7 \mathrm{~d}$ (min-max: 2-370; median: 25.5). The presence of intestinal atresia was the only factor correlated with prolonged parenteral nutrition, delayed time to full enteral intake, and more extended hospitalization. In this study, intestinal atresia was the primary predictive factor for severity of gastroschisis.

Surgical repair should be offered promptly, commonly on the first day after delivery to minimize infection risks. Flap closure is an alternative to fascial closure for gastroschisis. Youssef et al. [13] performed a systematic review and meta-analysis of outcomes comparing these surgical techniques. These investigators analyzed the following outcomes: mortality, ventilation days, feeding parameters, length of stay (LOS), wound infection, resource utilization, and umbilical hernia incidence. Twelve studies were included allowing evaluation of 1124 patients of which 350 underwent flap closure (210 immediately; 140 post-silo). Their meta-analysis revealed no significant differences in mortality, LOS, or feeding parameters between groups. Flap patients had fewer wound infections (OR=0.40; 95\% CL: 0.22-0.74; and $\mathrm{P}=0.003$ ). Flap patients had an increased risk of an umbilical hernia, but they were less likely to undergo repair (19\% vs. $41 \%$; P $=0.01$ ). These investigators concluded that flap closure has equivalent or superior outcomes to fascial closure for patients with gastroschisis and suggest that with its potential advantages of bedside closure and reduced sedation requirements; flap closure may represent the preferred closure strategy.

\section{Developmental factors and genomic MOAs}

Gastroschisis is a unique birth defect due to its association with young maternal age and increasing global prevalence. In the absence of evidence of a specific genetic cause or environmental teratogen, the underlying pathophysiological mechanisms are undefined. A thoughtful commentary by Opitz [14] considered whether gastroschisis is better characterized as a primary or secondary malformation.

Opitz profiled primary malformations as a "developpmental field defect" defined based on three cardinal criteria as follows:

- Heterogeneity; namely, the demonstration of causal heterogeneity of a malformation as evidence of identical reactivity to different endogenous causes on an embryonic primordium, common to all the clinical entities under discussion.

- Homology in that the embryonic primordia in humans capable of being malformed have the identical morphogenetic counterpart in more or less closely related mammals or vertebrates with corresponding molecular induction systems.
- Phylogeneity; from the concept of homology, it follows that corresponding anatomical structures in various species arising in response to more or less identical molecular induction cascades.

This is in contrast to a secondary malformation, which would be observed in an individual who was presumably genetically normal at conception but then experienced altered morphogenesis due to exposure to some exogenous stressor(s) such as chemical, physical, infectious, and maternal metabolic or psycho-social factors.

Some non-genetic factors have been implicated in the occurrence of gastroschisis, but no single factor stands out as a likely predominant cause. Drongowski et al. [15] reviewed the antenatal history of 19 infants with gastroschisis and 54 control infants born with a congenital anomaly unrelated to gastroschisis. When compared to controls, mothers of infants with gastroschisis were more likely to have used aspirin during pregnancy, to be taking oral contraceptives at the time of conception or to use an illegal drug, particularly cocaine.

Payne et al. [16] studied growth restriction in gastroschisis to consider if placental factors might be a cause. These investigators compared to birth weight (BW), crownheel length (LT), occipitofrontal circumference (OFC) and ponderal index (PI) in 179 gastroschisis cases and 895 matched controls. Fetal ultrasounds $(n=80)$ were reviewed to determine the onset of growth restriction and placental histology was examined in 31 gastroschisis patients and 29 controls. Gastroschisis cases weighed less than controls (BW, $2400 \pm 502$ g vs. $2750 \pm 532$ g; $<<0.001$ ) and they had lower BW as a group compared to controls. Intrauterine growth restriction was symmetric with gastroschisis patients having a shorter LT, smaller OFC but larger ponderal index compared to controls. Growth deficits appeared early in the second trimester and worsened as gestation increased. Placental chorangiosis was more common in gastroschisis patients than controls, even after removing all small for gestateonal age patients.

Mac Bird et al. [17] investigated associations between potential maternal factors with the risk of gastroschisis and omphalocele within a large population-based sample of participants enrolled in the National Birth Defects Prevention Study between October 1997 and December 2003. Data were collected on 485 cases of gastroschisis, 168 cases of omphalocele, and 4967 controls. These investigators found the expected higher risk in younger women and lower risk in Black women but also found a moderately increased risk of gastroschisis in women who had smoked tobacco, taken ibuprofen or consumed alcohol.

Duong et al. [18] compared mothers of infants with birth defects $(n=10,825)$ and mothers of infants without birth defects $(n=6795)$ who participated in the multisite National Birth Defects Prevention Study between 1997 and 2005. They found that mothers of infants with gastroschisis were significantly more likely to report any use of a hot tub in early pregnancy. Among the mothers who reported using a hot tub more than once in the exposure 
period and remaining in it for more than $30 \mathrm{~min}$, they found significantly elevated risk $(\mathrm{OR} \geq 2.0)$ for gastroschisis and some other birth defects.

In a related report, Agopian et al. [19] assessed the relationships between bathing habits during pregnancy and the risk of 13 non-syndromic birth defects in the National Birth Defects Prevention Study delivered during 20002007. These investigators found that the risk for gastroschisis in offspring was increased among women who reported showers lasting more than 15 compared to less than 15 minutes. It is at least plausible that this observation might relate to the duration of exposure to high water temperatures for somewhat extended episodes during early pregnancy.

Lin et al. [20] assessed maternal occupation and the risk of significant birth defects. For gastroschisis, an increased risk was found for maternal occupations of entertainers or athletes. In addition, they found a decreased risk of gastroschisis for the maternal occupation of being a non-preschool teacher.

Ortega-García et al. [21] raised the possibility of psychosocial stress as a potential risk factor for gastroschisis. In a case-control study of gastroschisis in Spain from December 2007 to June 2013, these investigators found that mothers of children with gastroschisis were younger, smoked more cigarettes, used more illegal drugs, and suffered domestic violence more frequently than the controls.

In 2015, Skarsgard et al. [22] reported on their investigation of the threefold increase in gastroschisis in Canada over the previous 10 years. They compared 692 gastroschisis pregnancies from the Canadian Pediatric Surgery Network and 4708 pregnancies from the Canadian Community Health Survey. Younger maternal age, smoking tobacco, a history of pregestational or gestational diabetes, and use of medication to treat depression all showed significant associations with increased risk of gastroschisis.

\section{Genetic, genomic, gene variants, epigenetics MOAs}

Bugge et al. [23] published the first case report on female monozygotic (MZ) twins discordant for gastroschisis. They found no family history of gastroschisis and no suspicious exposures during the pregnancy. Zygosity of the infants was verified by DNA analysis using highly polymorphic microsatellites. Albeit only one case, this observation does not support an explanation via a simple inherited gene etiology.

There is an inbred mouse strain (HLG) that shows a high incidence of gastroschisis after X-ray exposure of the zygotes with about $10 \%$ of fetuses having this malformation after irradiation with $1 \mathrm{~Gy}$. Assessment of data from crossbreeding studies [24] suggests that a single-locus inheritance is not a good fit, and two or three gene loci are likely to be involved. Remarkably, additional evidence suggests that the elevated risk of gastroschisis can be transmitted to the next mouse generation [25] and su- ggests that the induced genomic instability might be a factor in the known familial risk of gastroschisis.

In another mouse model of gastroschisis, for the AEBP1 (adipocyte enhancer binding protein) gene, isoform Aclp $(-/-)$ mice demonstrate a ventral wall defect that is similar to gastroschisis in humans [26].

Feldkamp et al. [26] reasoned that Aclp is a potential candidate gene for gastroschisis due to its developmental expression, association with the extracellular matrix and is essential for abdominal wall development. From this mechanistic perspective, Feldkamp et al. proceeded with assessing AEBP1 gene variants in 40 cases of infants with gastroschisis. These investigators identified several novel variants in AEBP1, but the frequency of these variants did not significantly differ between the cases and the control group. Additionally, they used bioinformatics programs to predict the likely amino acid changes, and these changes were predicted to be benign.

With the hypothesis that genes related to vascular compromise may interact with environmental factors to affect the risk of gastroschisis, Padula et al. [27] conducted a population-based case-control study of 228 infants in California. They evaluated 75 genetic variants in 20 genes and the risk of gastroschisis associated with maternal age, race or ethnicity, vitamin use, and tobacco smoking exposure. These investigators found 11 gene variants with increased risk and four variants with decreased risk of gastroschisis for heterozygous or homozygous variants genotypes and suggested that NOS3, ADD1, ICAM1, ICAM4, and ICAM5 warrant further investigation and may interact with environmental exposures.

Makhmudi et al. [28] sought to assess the hypothesized dual vascular/thrombotic pathogenesis of gastroschisis in which it is argued that normal right umbilical vein involution forms a possible site for thrombosis adjacent to the umbilical ring. Accordingly, these investigators measured the frequency of three common prothrombotic polymerphisms, MTHFR c.677C>T, F2 c.20210G>A, and F5 Leiden in 46 Indonesian gastroschisis patients and in 89 ethnicity-matched controls. While MTHFR c. $677 \mathrm{C}>\mathrm{T}$ showed a significant association with gastroschisis (OR= 2.1), no affected infants had risk alleles for either F2 c. $20210 \mathrm{G}>\mathrm{A}$ or F5 Leiden. This finding lends support to the thrombotic pathogenesis hypothesis for gastroschisis.

As a robust alternative to the vascular pathogenesis hyp-othesis for the occurrence of gastroschisis, an umbilical ring defect theory has been proposed, based on embryological studies. [29; 30] The embryological argument is that if a vascular insult to the vitelline artery were to be the proximate cause of gastroschisis, then the entire midgut should be injured rather than the abdominal wall, which gets its vascular supply from the intersegmental arteries. In turn, if insults to these vessels caused gastroschisis, then there is no explanation for the preponderance of gastroschisis occurring on the right rather than equally on the left. If the occurrence of gastroschisis does indeed derive from disordered development or inadequate maintenance of the structural integrity of the umbilical 
ring and its amnion-epithelial connection, then cellular and molecular systems that regulate tissue organization, cellular signaling, and movements while maintaining the structural integrity of adjacent tissues should be evaluated.

\section{RISK FACTORS}

In 1990, Goldbaum et al. [31] reported on their assessment of risk factors that might explain an observed increase in gastroschisis in residents of Washington State during the interval 1984-1987. They reviewed birth certificates for 62 infants born with gastroschisis and 617 randomly selected unaffected infants matched for birth year. The four risk factors that "stood out" were a birth month (higher risk in January, February, and March); mothers age younger than 25 years and especially age younger than 20 years; tobacco smoking during pregnancy; and mothers receiving inadequate prenatal care. These investigators suggest that it is likely that other unidentified behaviors and environmental exposures could explain the risk associations with birth month, young maternal age, and limited prenatal care.

In a different U.S. region, Chabra \& Hall [32] assessed a single center cluster of 10 cases of gastroschisis at the Neonatal Intensive Care Unit at the University of Kentucky Medical Center in 1996. These investigators then retrospectively sought environmental or genetic causes. They reviewed the maternal and patient medical records for 36 neonates with gastroschisis admitted from January 1992 to December 1996. While there was evidence that many of the mothers were teenagers, primiparous, and had an increased frequency of tobacco smoking, they found no evidence of temporal or spatial clustering in the gastroschisis cases.

In an excellent comprehensive review about a decade ago, Rasmussen and Frias [33] summarized what was known about non-genetic risk factors for gastroschisis. These authors highlighted several key observations that in turn suggested directions for future research opportunities. With some degree of disappointment, research needs mostly remain current and we hope to suggest some new directions that merit attention.

Rasmussen and Frias noted that the etiology of gastroschisis is unknown, but the familial increased risk (approximately $3.5 \%$ ) in the families with a previous child with gastroschisis suggested that genetic factors play a role in its causation. While that genetic relationship is logical, it is also true that cohabiting family members share many of the same environmental exposures, so the same reasoning could apply to support the notion that a familial pattern of risk may be attributable to sharing a common multifactorial "risky" environment.

Beyond the widely demonstrated risk of younger maternal age, other robust individual exogenous risk factors are not as consistently found. There is some evidence that exposures such as sociodemographic factors, maternal medication use, substance use/abuse, workplace or environmental chemical exposures do correlate with some increased risk of gastroschisis. Since a prevailing hypo- thesis is that gastroschisis is often due to some vascular disruption during early development, it can be argued that all of these are candidates for clinical or public health protective or mitigative interventions. For most of these exposures, it is reasonable to suppose that gene-environment interactions are key to manifestation of the outcome of gastroschisis, and there is some evidence that this notion is applicable to the risk factor of maternal tobacco smoking and the occurrence of variant alleles of genes such as NOS3, NPPA, and ICAM1 involved in the VEGF-NOS3 pathway [34].

\section{Maternal illnesses, medication use, and substance abuse}

Several groups of investigators have studied the possible impact of several exogenous factors in the risk of gastroschisis. Quite logically the efforts have focused on maternal exposures in the periconception and early gestational intervals when key embryonic developmental events might be influenced in a cause and effect manner. Several factors, including maternal demographics, medical illnesses, environmental exposures, use of prescribed and over-the-counter medications, consumption of ethanol, and use of recreational drugs, have been assessed to some extent in the risk of gastroschisis.

Werler et al. [53] analyzed data from a case-control surveillance program of birth defects from 1976-1990. They compared maternal demographic, reproductive, and medical factors and first-trimester environmental expoures between 76 gastroschisis cases and 2,581 malformed controls. They found the expected strong inverse association with maternal age but also detected a relationship of increased risk of gastroschisis with maternal ethanol consumption, roughly doubling or trip-ling the risk.

In another study of multiple possible risk factors for gastroschisis, Torfs et al.[36] reported on several associations for maternal medications and environmental exposures as follows:

- Hobby or occupational exposures to solvents (odds ratio $(\mathrm{OR})=3.8$ or colorants $(\mathrm{OR}=2.30)$;

- Use of medications aspirin (OR=4.7) and ibuprofen $(\mathrm{OR}=4.0)$ but not for acetaminophen;

- $\quad$ Periconceptional exposure to X rays $(\mathrm{OR}=2.5)$;

- Use of antibiotics, antinauseants, sulfonamides, or oral contraceptives were not associated;

- Use of decongestants, pseudoephedrine (OR=2.1), phenylpropanolamine $(\mathrm{OR}=10.0)$, group of all decongestants including oxymetazoline and ephedrine $(\mathrm{OR}=2.4)$.

From these observations, the authors remarked that since most of these associations were for vasoactive substaances, their results support a vascular hypothesis for the pathogenesis of gastroschisis.

Carrying forward the idea of vascular disruption as a critical component in the events that may lead to gastroschisis, Hume et al. [37] considered the potential effect of prenatal cocaine exposure in gastroschisis. These investigators performed a retrospective case-control study 
based upon more than 68,000 delivery records at a single hospital for 9 years. Transverse limb defects and gastroschisis were defined as cases, and non-disruption anomalies served as controls. In 190 cases of limb anomalies, abdominal wall defects, and cleft lips, 119 cases had information regarding maternal cocaine use during pregnancy. Hume et al. found 7 of 34 vascular disruption cases associated with cocaine exposure versus 12 of 85 other malformations controls yielding an odds ratio for cocaine exposure and vascular disruption of 1.58 (95\% CL: 0.55-4.47). Although there are limitations to these data, these results did not seem to support the idea that prenatal cocaine exposure might influence gastroschisis risk via a vascular disruption effect.

A few reports suggest an association between other drugs of abuse and the occurrence of gastroschisis. In two studies by one group of investigators [38; 39], maternal exposures to recreational drugs were ascertained by measurement of individual recreational drugs in maternal hair samples timed for the period of conception and in different stages of pregnancy in expectant mothers with a diagnosis of fetal gastroschisis and in a group of wowen with a normal fetus as controls. Overall, these investigators found evidence of recreational drug use in $18 \%$ of women with fetal gastroschisis and confirmed the association of gastroschisis with young maternal age. In another recent report, a higher prevalence of gastroschisis was found in regions with the US where rates of opioid prescriptions were highly indicative of an association between opioid use during pregnancy and gastroschisis. [40]

While most reports on gastroschisis derive from clinical investigations, Burdan et al. [41] have presented data in an animal model to compare the effects of drugs within a single category, namely cyclooxygenase inhibitors. The effects of prenatal exposure to various selective and nonselective cyclooxygenase-2 (COX-2) inhibitors on abdominal wall defects in the rat were assessed by a retrospective analysis of laboratory data from several teratological studies with COX-inhibitors (aspirin, DFU, DuP-697, ibuprofen, paracetamol, piroxicam, propyphenazone, tolmetin) throughout 1997-2004. In 6744 live-born fetuses, abdominal wall defects were found in four animals exposed to different non-selective COX inhibitors and one case of gastroschisis in a rat exposed to a selective COX-2 inhibitor. In their analysis of the various drugs, only aspirin statistically increased the risk of abdominal wall defects in rat fetuses with an expected ratio for aspirin of 56 per 10,000 offspring.

A cluster of gastroschisis cases $(n=14)$ was observed in April 2007- April 2008 in Reno, Nevada. Elliott et al. [42] performed a case-control study to identify potential risk factors. In comparison to controls ( $n=57$; matched 4:1 to the case mothers by maternal date of birth within 1 year) , gastroschisis was associated with the prepregnancy use of methamphetamine (OR=7.15) or any vasoconstrictive recreational drugs (methamphetamine, amphetamine, cocaine, ecstasy) with OR of 4.46. These findings support the notion that use of vasoconstrictive recreational drugs is a risk factor for gastroschisis.

The commonly used drugs of alcohol (ethanol), tobacco, and cannabis have also been implicated as risk factors for gastroschisis. First, in the large multicenter National Birth Defects Prevention Study (6622 control infants and 1768 infants with birth defects born in 1997-2005), Richardson et al. [43] performed a case-control study to assess any association between periconceptional alcohol consumption and several birth defects including omphalocele and gastroschisis. These investigators found that periconceptional alcohol consumption was associated with omphalocele $(\mathrm{OR}=1.50 ; 1.15-1.96)$ and gastroschisis (OR=1.40; 95\% CL: 1.17-1.67). Second, as regards maternal smoking, Hackshaw et al. [44] conducted a meta-analysis using data from 172 articles with a total of 173,687 malformed cases and 11,674,332 unaffected controls and reported a significant positive association of maternal smoking with gastroschisis $(\mathrm{OR}=1.50,95 \%$ CL:1.28-1.76). Additionally, it has been hypothesized that differences in genetic susceptibility may exist regarding this association of smoking with gastroschisis. In one study that investigated this possibility, Jenkins et al. [45] analyzed five single nucleotide polymorphisms in three genes (CYP1A1, CYP1A2, and NAT2) that encode for enzymes involved in metabolism of some cigarette smoke constituents in 108 non-Hispanic white and 62 Hispanic families who had infants with gastroschisis, and 1,147 non-Hispanic white and 337 Hispanic families who had liveborn infants with no significant structural birth defects (controls). While these investigators did identify three suggestive associations among 30 potential associations, they concluded that these data did not support the occurrence of effect modification between periconceptional maternal smoking and most of the xenobiotic metabolizing enzyme gene variants assessed. Third, using multiple logistic regression, van Gelder et al. [46] reanalyzed associations between periconceptional cannabis use and 20 specific birth defects using data from the National Birth Defects Prevention Study from 19972005 for 13,859 case infants and 6,556 control infants and found that after correction for exposure misclassification, cannabis use was associated with gastroschisis with an OR of 1.7 .

A modest number of prescription medications have also been implicated as risk factors for gastroschisis when mothers have used such drugs at various intervals from preconception through approximately the third month of pregnancy. It must be noted that any association of gastroschisis risk may be due either to a) the underlying maternal disease for which a drug was prescribed, b) use of the drug per se or c) the co-exposure of the embryo/ fetus to the maternal disease and the drug in question. Nonetheless, the drugs for which there is some evidence of an associated risk include venlafaxine [47], antiherpetic medications (acyclovir, valacyclovir or famciclovir) [48], levonorgestrel/ethinylestradiol [49], antidepressants [50], topical antivirals [50], and continuation of oral contraceptives in early pregnancy [50]. As noted by Given et al. [50], "While it is difficult to disentangle the 
effects of the medication and underlying indication, our results add to the evidence base on preventable risk factors for gastroschisis. These risk factors may contribute to the higher risk among young mothers, and geographical and temporal variation in prevalence." Additionally, another infectious agent, chlamydia trachomatis, has been suggested by Feldkamp et al. [51] as a risk factor for gastroschisis based on observation of the unusual finding of vacuolated amniotic epithelium with lipid droplets in gastroschisis placentas, combined with some experimental evidence of the trafficking of host lipids into the chlamydia intracellular inclusions.

\section{Maternal nutrition and metabolic milieu}

In order to diminish the risk and/or severity of gastroschisis, other than identifying and limiting exposures to possible exogenous hazards, endogenous maternal factors that could be favorably modified should be considered. These factors, which include various exogenous-endogeenous (gene-environment) interactions, encompass at least metabolic, nutritional, vascular, and inflammatory components.

In a past animal study of gastroschisis, interactions of nutritional factors and a smoking-related component were studied. Singh [52] maintained pregnant CD-1 mice on diets with two levels of protein and three levels of zinc, and exposed half of each diet subgroup to either air (control) or to 500 ppm carbon monoxide (CO) from gestation days (GD) 8-18. At necropsy, fetal mortality and malformations were increased by protein and zinc deficiencies, and CO exposure increased fetal mortality. The incidence of gastroschisis in the low protein/zinc deficient/CO exposed group was $47 \%$, and $60 \%$ of the litters were affected. The incidence of gastroschisis in the rest of the low protein/zinc diets/air or CO groups was zero. In this model, gastroschisis is caused by the combination of protein-zinc deficiencies plus CO exposure during gestation and thus may be relevant to human populations who may have nutritional deficiencies and exposure to $\mathrm{CO}$ via environmental or maternal smoking (tobacco or cannabis).

In another past study of 57 cases of gastroschisis and 506 controls, Torfs et al. [53] tested for DNA polymorphisms of 32 genes representing enzymes involved in angiogenesis, blood vessel integrity, inflammation, wound repair, and dermal or epidermal strength. These investigators found that several gene polymorphisms were associated with an increased risk for gastroschisis for heterozygotes (ICAM1 gly241arg; NOS3 glu298asp; NPPA 2238 T>C; and ADD1 gly460trp) and that for the NPPA and ADD1 single-nucleotide polymorphisms (SNPs), the homozygote variants had a significantly higher risk than the heterozygotes. Additionally, three SNPs (NOS3; ICAM1; and NPP) showed a strong interaction for risk with maternal smoking, supporting the hypothesis that gene-environmental interactions are a part of the vascular compromise that is plausibly involved in the etiology of gastroschisis.
Maternal nutrition and either low maternal body mass or maternal obesity have been considered in some studies of gastroschisis. Waller et al. [54] assessed the relationship between maternal obesity, overweight and underweight status, and 16 categories of structural birth defects in mothers enrolled in the National Birth Defects Prevention Study who had index pregnancies between October 1, 1997, and December 31, 2002. These investigators found that mothers of offspring with omphalocele were significantly more likely to be obese than mothers of controls (ORs between 1.33 and 2.10) while mothers of offspring with gastroschisis were significantly less likely to be obese than mothers of controls. These results suggested a strong inverse association of obesity with gastroschisis. In another study, Siega-Riz et al. [55] evaluated the joint effects of maternal pre-pregnancy body mass index and maternal age on the risk of gastroschisis. These investigators reported that younger women who had lower BMI were at highest risk of having an infant with gastroschisis. For example, a 15-year-old woman with a BMI of 17 has seven times the odds of having an offspring with gastroschisis compared with a 24-yearold woman with a BMI of 23. Finally, Paranjothy et al. [56] emphasized the importance of maternal nutrition in the etiology of gastroschisis in their study. These investigators assessed high maternal alcohol consumption and poor diet in the first trimester as risk factors in a casecontrol study in the UK. Their results showed that high consumption of fruits and vegetables during the first trimester $(\mathrm{OR}=0.2)$, taking folic acid for at least 6 weeks during the first trimester $(\mathrm{OR}=0.3)$ and increased body fat percentage of total maternal body weight $(\mathrm{OR}=0.9)$ were independently associated with reduced risk and that cigarette smoking $(\mathrm{OR}=2.7)$ was an independent factor for increased risk.

In summary, beyond the apparent need to reduce or avoid smoking by women in the prepregnancy and gestational intervals, interventions to attain better nutritional status in terms of both diet quality (intake of fruits and vegetables) and adequacy of caloric intake particularly for younger gravidas, must be part of the public health effort to reduce the risk of gastroschisis in infants.

\section{Environmental exposures}

One general hypothesis for any disease showing an increase over time is that changing environmental, dietary or occupational exposures might be a causal factor. Therefore, a modest number of studies have tried to determine if various exposures from those sources might be implicated in gastroschisis.

A few studies have assessed the risk of various birth defects including gastroschisis in populations residing near hazardous waste landfill sites. In the EUROHAZCON study, Dolk et al. [57] studied the risk of congenital anomalies near hazardous-waste landfill sites in Europe. For overall congenital anomalies, residence within $3 \mathrm{~km}$ of a landfill site was associated with a significantly raised risk (295 cases/511 controls living 0-3 km from sites, 794/ 1855 living 3-7 km from sites) with an $\mathrm{OR}=1.33$. For gastroschisis alone, the risk was only borderline signifi- 
cant with $\mathrm{OR}=3.19$ [0.95-10.77]). In another study in the UK, Fielder et al. [58] studied the health of residents living near the Nanty-Gwyddon landfill site. While their neonatal data showed some complexity, they reported that there was no consistent difference in the proportion of low birth-weight infants before and after the opening of the landfill site. Additionally, among resident living near the site, there was an increased risk of newborns having a congenital abnormality, but this was seen both before (relative risk, $\mathrm{RR}=1.9$; 95\% CL: 1.3-2.85; $\mathrm{P}<0.001$ ) and after (RR=1.9; 95\% CL: 1.23-2.95; $\mathrm{P}=0.003)$ its opening. However, they noted that an observed cluster of cases of gastroschisis was seen only after opening of the site. Finally, a broader study in the UK also assessed the risk of adverse birth outcomes in populations living near landfill sites. Elliott et al. [59] identified populations living within $2 \mathrm{~km}$ of 9,565 landfill sites operational at some time between 1982 and 1997 and included more than 8.2 million live births, 43,471 still-births, and 124,597 congenital anomalies (including terminations). For all anomalies combined, the relative risk of a residence near landfill sites was not convincingly associated with risk (slight decrease for unadjusted $\mathrm{OR}=0.92$; slight increase for adjusted $\mathrm{OR}=1.01$ ). However, for abdominal wall defects and surgical correction of gastroschisis and omphalocele, they found adjusted risks with $\mathrm{OR}=1.08$ (95\% CL: 1.01-1.15) and OR=1.19 (95\% CL: 1.05-1.34) respectively. In summary, if there is any association of residence near waste landfill sites and gastroschisis, then the association is quite weak. The strength of evidence does not support a public health advisory to relocate residences away from such landfill sites to mitigate the risk of gastroschisis.

Another hypothesized route of exposure that might influence gastroschisis risk is drinking water. One study [60] in North Carolina considered this possibility. Root and Emch traced drinking water to its sources and how those sources related to the locations of textile mills. These investigators found no association between prenatal exposure to upstream textile mill effluent and risk of gastroschisis; however, they did report an increased risk in women who relied on public water systems that drew from a surface water source rather than a ground-water source.

Seemingly, limited consideration has been given to possible maternal occupational exposures as a risk factor for gastroschisis. In a single report, Lupo et al. [61] studied maternal occupational exposure to polycyclic aromatic hydrocarbons (PAHs) in the National Birth Defects Prevention Study. In this large data set, the prevalence of estimated occupational PAH exposure was $9.0 \%$ in case mothers (27 of 299) compared to $3.6 \%$ in control mothers (107 of 2993). Remarkably, they found a significant association between occupational PAHs and gastroschisis among mothers at or older than 20 years of age (adjusted $\mathrm{OR}=2.53$; 95\% CL: 1.27-5.04); however, they did not find such an association in mothers younger than 20 years (adjusted OR=1.14; 95\% CL: 0.55-2.33). The investigators noted that this observation might be meaningful since, on the one hand, young maternal age is the most potent known risk factor for gastroschisis while on the other hand, most gastroschisis cases are born to mothers at age of more than 20 years.

The plausibility of dietary or environmental chemicals as teratogens that can cause congenital abdominal wall defects including gastroschisis is generally supported by studies in animal models $[62 ; 60]$. In attempting to translate/correlate data from animal models with observations in human populations, at least one caution is waranted. There may be discrepancies in the nomenclature used by laboratory teratologists and that used by physicians and epidemiologists. These differences may substantially matter since evidence broadly suggests that in human populations, rates of omphalocele have not changed while gastroschisis rates have increased. With this provision, compounds that have been implicated in animal models include mycotoxins [62] as well as a wide range of other compounds, reported by van Dorp et al. [63] to include induction of umbilical cord hernia by 8 , omphalocele by 9 and gastroschisis by 22 teratogens.

\section{Distinct exposure category - agricultural chemicals}

One distinct category of potential chemical exposures that can be distinguished by possibly having shared occupational, residential and/or dietary routes of exposure is agricultural chemicals such as herbicides, fertilizers, fungicides, pesticides, and petroleum products. Indeed, a few reports suggest that some such exposures may be risk factors for gastroschisis.

Waller et al. [64] conducted a retrospective, case-control study using Washington State Birth Certificate and US Geological Survey databases in 805 cases defined as all live-born singleton infants with gastroschisis and 3616 controls. Gastroschisis occurred more frequently among those who resided $25 \mathrm{~km}$ from a site of high atrazine concentration $(\mathrm{OR}=1.6)$. The risk was related inversely to the distance between the maternal residence and the closest toxic atrazine site. In multivariate analysis, nulliparity, tobacco use, and spring conception were significant predictive factors for gastroschisis. Based on these data, these investigators remarked "maternal exposure to surface water atrazine is associated with fetal gastroschisis, particularly in spring conceptions." In a subsequent study conducted in Texas using similar study techniques, Agopian et al. [65] evaluated the relationship between maternal atrazine exposure and gastroschisis risk by maternal age in 1,161 gastroschisis cases and 8,390 controls. In this latter study [65], there was no association of maternal atrazine exposure and gastroschisis risk in women under 25 years of age; however, there was an increased risk for gastroschisis in offspring of women at or older than 25 years with counties of residence that had higher levels of potential residential atrazine exposure. These investigators noted that these results suggest that the etiology of gastroschisis may vary based on maternal age.

In an interesting study in an animal model, the possibility of a derivative of two different classes of agricultural chemicals (atrazine and nitrates) was assessed for inducti- 
on of embryonic malformations. Given the findings cited above about atrazine as a possible risk factor for gastroschisis, consider as well that the U.S. Geological Survey states on its website [The USGS Water Science School; https://water.usgs.gov/edu/nitrogen.html] "Nitrate can get into the water directly as the result of runoff of fertilizers containing nitrate." Thus, Joshi et al. [66] studied the effects of developmental exposure of the chicken to the potential reaction product of nitrate and atrazine $\mathrm{N}$-nitrosoatrazine (NNAT). These investigators treated fertilized eggs with 0.06-3.63 $\mu$ g NNAT and continued incubation for five more days. With 90\% survival, 23\% of embryos were malformed. Multiple malformations were documented, among these gastroschisis.

Finally, two investigations have attempted to take a broader approach to assess the potential risk of gastroschisis in association with exposures to various multiple agricultural chemicals. Using data from the National Birth Defects Prevention Study among employed women, Kielb et al. [67] conducted a multi-site case-control analysis that included the following designations: any occupational exposure (yes/no) to pesticides, to insectcides only, to both insecticides and herbicides $(\mathrm{I}+\mathrm{H})$ and to insecticides, herbicides and fungicides $(\mathrm{I}+\mathrm{H}+\mathrm{F})$. The data showed that occupational exposure to $\mathrm{I}+\mathrm{H}+\mathrm{F}$ was associated with the risk of gastroschisis among infants of women at or older than 20 years $(\mathrm{OR}=1.88)$ but not for women younger than 20 years of age. Shaw et al. [68] studied 156 cases (infants/ fetuses with gastroschisis) and 785 controls (infants without birth defects) regarding early pregnancy agricultural pesticide exposures and risk of gastroschisis in the San Joaquin Valley of California. The investigators analyzed associations of gastroschisis with 22 chemical pesticide groups and 36 specific pesticide chemicals. No association was found with any of the pesticide groups, and among the specific pesticide chemicals, only exposure to petroleum distillates was associated with an elevated risk (OR=2.5; 95\% CL: 1.15.6).

In summary, there are some data suggesting associations between exposure to a few agricultural chemicals and risk of gastroschisis. As a general public health approach, it would be prudent to minimize exposure of women prepregnancy and during early weeks/months of gestation to commonly used agricultural chemicals for which there is some concerning data.

\section{POTENTIAL MOA HYPOTHESES}

A few mode-of-action (MOA) hypotheses have been proposed as explanations for associations of gastroschisis with various exposures. As cited earlier in this article, several investigators have argued that a vascular compromise MOA for gastroschisis seems to cogently link several observations $[27 ; 28 ; 33 ; 34 ; 36 ; 37 ; 53]$.

Other plausible hypotheses for gastroschisis were reviewed and two new hypotheses were advanced in back-toback publications $[69 ; 70]$ a few years ago. Feldkamp M et al. [69] carefully considered existing alternative embr- yonic hypotheses for the occurrence of gastroschisis as follows:

a) Failure of mesoderm to form in the body wall;

b) Rupture of the amnion around the umbilical ring with subsequent herniation of bowel;

c) Abnormal involution of the right umbilical vein leading to weakening of the body wall and gut herniation; and

d) Disruption of the right vitelline (yolk sac) artery with subsequent body wall damage and gut herniation.

e) These investigators commented that in their view, that these hypotheses were not adequate to explain how gastroschisis could occur, and thus proposed an alternative embryonic hypothesis; namely,

f) Abnormal folding of the body wall results in a ventral body wall defect through which the gut herniates, leading to the clinical presentation of gastroschisis.

In the second of these two publications, Chambers et al. [70] described conduct of a case-control study to compare the prevalence of change in paternity with the index pregnancy in 102 mothers of gastroschisis cases to that in 117 mothers of non-malformed infants and in 78 mothers of infants with neural tube defects or oral clefts. After adjustment for maternal age, change in paternity in multigravid gastroschisis case mothers was higher (OR =7.81; 95\% CL: $2.80-21.88$ ) relative to multigravid mothers of malformed and non-malformed controls combined. Based on these results, the investigators suggest support for an additional hypothesis that maternal immune factors may play a causative role in gastroschisis.

These several hypothesized MOAs are plausible but do not offer the degree of detail about cellular or molecular processes, signaling cascades and/or feedback (dys) regulation that is fully satisfying for a deep understanding of the basis for the occurrence of this malformation. Additionally, at present these hypotheses do not suggest potential protective (preventive or mitigative) interventions other than the general notion of avoidance of suspect exposures. To take meaningful translational toxicology/teratology steps and strive toward identifying one or more safe and efficacious protective nutritional, lifestyle or pharmaceutical therapies, new cellular/ molecular MOAs need to be considered and investigated. We suggest three as follows:

a) most tentatively, changes in epigenetic programing of relevant stem or progenitor cells;

b) mechanical forces MOA meaning the mechanobiology of collective cell behaviors; and

c) ephrin-Eph signaling as a developmental MOA.

As a dynamic field of study, epigenetics is seemingly involved in or relevant to almost every area of biological regulation. We may have overlooked some reports, but we have not seen published evidence suggesting that epigenetic programming has been explicitly investigated in gastroschisis. To illustrate the range of potential effects that epigenetic mediation of exposures could exert, consider the comments of Bateman et al. in a recent review 
[71]. These authors noted that several environmental chemicals often-called endocrine disrupting chemicals (EDCs) might induce the changes in mesenchymal stem cells (MSCs). Such actions may include alterations in adipogenic differentiation, osteogenic differentiation, activation of pro-inflammatory cytokines, oxidative stress, trophic factor production, the immune-modulatory capacity of MSCs, differentiation into appropriate cellular lineages, and paracrine signaling in wound healing. Several of these effects could be a clue with relevance to basic mechanisms by which gastroschisis occurs, at least in some cases.

\section{MOA - mechanical forces - mechanosensitivity and mechanotransduction signaling}

A potentially robust MOA underlying gastroschisis could be in the mechanobiology of collective cell behaviors [72; $73 ; 74 ; 75 ; 76 ; 77]$. Cells and cell collectives respond to multiple non-mechanical signals and gradients such as gradients of diffusible molecules and electrical fields but also mechanical signals. Cells sense chemical and mechanical signals in their local microenvironment and both classes of signals regulate gene expression programs in the nucleus. With consideration of possible disordered signaling in the occurrence of gastroschisis, the fates of cells and cell collectives in development are subject to mechanical and morphological cues that function as critical signaling mechanisms. Ligands provide cues in the extracellular matrix (ECM), but also physical properties including ECM stiffness, cell shape, cell-substrate adhesion, cell-cell adhesions, and cytoskeleton architecture all of which inform cells and cellular collectives of their respective surrounding locale.

Mechanical properties of the external environment influence the coordinated behaviors of cells during key biological processes such as morphogenesis and tissue remodeling $[72 ; 73 ; 74 ; 75 ; 76 ; 77]$. Collections of cells interact with both the surrounding extra-cellular matrix and with neighboring cells. The behavior of cellular collectives depends upon active interactions among cells to affect their movements. Mechanosensitive adhesion complexes regulate such collective movements at the cell-substrate interface as well as cell-cell junctions. Both types of conections permit cellular responses but also propagate physical signals. From Vining and Mooney [75], "Mechanical forces regulate cell fate decisions during organogenesis as progenitor cells are directed to diverse specialized functions in fetal organs.

Complex patterning depends on cell-ECM interactions. Biochemical cues initiate morphogenesis, but the formation of cell layers that become organized into defined structures in organs requires physical traction forces on the ECM, the physical properties of which provide a template for organ growth.

The concerted action of biochemical signals, cell-intrinsic forces, and cell-ECM interactions result in highly organized patterns of development, such as fractal patterns observed in branching morphogenesis. As development progresses, intrinsic forces exerted by cells transition from largely cell-cell to more cell-extracellular matrix (ECM) transmission because of matrix content in tissues increases."

There is a growing understanding of the mechanobiology of collective cell movements. Single cells use actomyosin contractility to exert traction forces on the extracellular matrix (ECM) at integrin-based adhesions. Adhesion complexes play a role in mechanosensitivity and mechanotransduction signaling in collective cell behaveiors. Additionally, various physical properties of the cellular environment can regulate collective cell behaviors, tissue organization and cell-generated forces that encompass molecular, cellular and tissue levels. Single cells and cells migrating in cell collectives polarize by extending lamellipodia at their contact with ECM or with other cells at the boundary. On the scale of collective cell movements, individual cells may exert traction on either the ECM or on neighboring cells.

Cell division and extrusion also alter tissue movement and contribute to the propagation of strain and velocity waves driven by mechanobiochemical signals. Cells on a leading edge also form focal adhesions.

Focal adhesions depend upon some key functional proteins that regulate mechanical coupling of cells. A prominent group consists of the cadherins including epithetlial (E)-cadherin, neuronal (N)-cadherin, placental (P)cadherin and vascular-endothelial (VE)-cadherin as well as cadherin 6 , cadherin 7 and cadherin 11 . The junctional protein afadin interacts with nectins in adherens junctions, which seem to support tissue integrity during collective cell migration and morphogenesis $[75 ; 76 ; 77 ; 78$; 79]. Additionally, zonula occludens proteins ZO1, ZO2, and $\mathrm{ZO3}$, desmosomes and intermediary filaments, in tight junctions also appear to contribute to intercellular mechano-coupling [80].

From Ladoux and Mege [72], "Substrate geometry influences the mode of collective migration. In areas of positive curvature (for example, the tips of finger-like structures pointing into the gap), cells predominantly move by active crawling. In areas of negative curvature (that is, where the gap bows into the tissue), prominent actomyosin cables are formed. Actomyosin contractility and active cell crawling operate additively in gap closure, leading to faster tissue velocity in regions of negative curvature. Cellular coordination, which is at the basis of various phenomena, most prominently including tissue shaping during morphogenesis, is a mechanoregulated, multiscale process integrating events on the molecular, cellular and multicellular scales that can be observed at a wide range of timescales, from milliseconds to days. Cellular mechanosensitivity and mechanotransduction signaling is intimately integrated with transcriptional programming, epigenetic modifications, and biochemical differentiation. Together these signals interact to influence cell fates. Tatapudy et al. [73] have summarized interrelationships among metabolism, reactive oxygen species (ROS), intracellular $\mathrm{pH}$ ( $\mathrm{pHi}$ ), and cell morphology as follows: 
"Metabolic inputs regulate epigenetics and cell signaling to promote changes in cell fate. Glycolysis produces metabolic intermediates that feed into the folate and one carbon metabolism cycle to

a) Produce S-adenosylmethionine (SAM), which is a cofactor for DNA methyl-transferases (DNMTs) and histone methyl-transferases (HMTs).

b) Glucose-derived acetyl-CoA enters the tricarboxylic acid (TCA) cycle to form citrate, which can be converted back to acetyl-CoA by ATP-citrate lyase. This source of acetyl-CoA (but not acetyl-CoA derived from fatty acid oxidation) contributes to the pool of nuclear acetyl-CoA that is essential for histone acetylation by histone acetyl-transferases (HATs).

c) a-ketoglutarate $(\mathrm{a}-\mathrm{KG})$, which is produced in the TCA cycle and the cytoplasm, is an essential cofactor for TET and Jumonji C enzymes, which demethylate DNA and histones, respectively.

Increased oxidative phosphorylation also generates reactive oxygen species (ROS), which promote oxidation, carbonylation, and hydroxylation as well as increase the levels of JNK and p38/MAPK pathway activity."

The three-dimensional organization of chromosomes in the nucleus regulates gene expression patterns, and chromosomal organization is regulated by nuclear mechano-transduction [74]. These signals are sensed and transduced in the nucleus through the cytoskeleton, and its constituents of actin, microtubules and regulatory molecules wherein actin exerts contraction and microtubules exert compression. This regulatory relationship has been hypothesized by Uhler and Shivashankar [74] as follows: "...mechanosensing of the extracellular signals from the microenvironment results in both activation of specific transcription factors and modulation of the cytoskeleton-nucleus links, leading to the arrangement of a particular chromosome and gene neighborhoods. The particular chromatin spatial configurations and post-translational modifications are important for guiding transcripttion factors to their target genes and obtaining optimal transcriptional outputs to maintain cellular homeostasis."

Mechanical coupling enables stem cells to respond to their local environment and to store information over time [75]. For example, changes in ECM induced by cells early in development can mechanically trigger changes in interacting cells at a later stage. These interactions regulate cell behavior and influence cell fates in development both in that locale and more distantly. In addition to intrinsic cell-generated forces, extrinsic shear, tension, and compression forces can be sensed by signaling molecules such as ion channels, modified receptor-ligand and mechanical changes in the cytoskeleton.

Mechanosensitivity signaling primarily depends upon cell-cell adhesion complexes in early development. As progenitor cells differentiate later in development, they produce and adhere to ECM. As the ECM content of tissues increases during development, cellular mechano-sensing also increases allowing cells to respond to changes in E$\mathrm{CM}$ characteristics such as stiffness, adjacent deposition, and local degradation.

\section{MOA - Ephrin-Eph rceptor signaling}

Eph proteins are transmembrane Tyr kinase receptors that interact with ephrins, which are membrane-tethered ligands. Eph receptors are grouped into EphA or EphB subfamilies depending on whether they preferentially bind to ephrin-As (membrane-anchored) or ephrin-Bs ( transmembrane ephrin ligands). This system provides short-distance cell-cell signaling that affects the cellular cytoskeleton, leading to cell-cell repulsion and sometimes cell-cell adhesion. This ephrin-Eph receptor mechanism appears to play an important role in pattern formation and morphogenesis by influencing cell sorting and positioning during developpment [84]. Since short-distance signaling between neighboring cells can be mediated by Eph receptors and ephrin ligands, this system can direct cell repulsion, cell-cell adhesion, cell proliferation, tissue boundary formation, and cell migration. Regarding possible relevance to the occurrence of gastroschisis, ephrin-Eph signaling regulates developmental cell sorting at tissue compartment boundaries which is plausibly important in normal abdominal wall formation.

Eph receptor-ephrin signaling is multimodal [84]. While Eph proteins behave as classical receptors and ephrins as their ligands, signaling also occurs in the counterdirection with Eph receptor proteins acting as ligands for the respective ephrins. This evokes simultaneous bidirectional intracellular signals in the respective cells. The complex pattern of Eph-ephrin signaling can be sumarized as follows:

a) Forward signaling - signal transduction goes from ephrins to Eph receptors;

b) Reverse signaling - signal transduction goes from Eph receptors to ephrins;

c) Bidirectional signaling - signal transduction simultaneously activates downstream pathways for both Eph receptors and ephrins;

d) Parallel signaling - signal transduction in which Eph receptors and ephrins on the same cell; activate in response to their respective ephrins and Eph receptors on a neighboring cell;

e) Anti-parallel signaling - signal transduction in which there is simultaneous ephrin-Eph receptor forward signaling but in counter directions.

Regarding molecular behavior on cell surfaces, Eph receptor-ephrin signaling depends upon interactions as multimers in signaling clusters. These signaling clusters appear to be important in cellular boundary formation during embryonic development. On the one hand, some embryonic boundaries are unstable and thus permit cell movements, but later stable boundaries allow segmentation of groups of cells and establishment of distinct tissues. A balance of adhesive and repulsive forces permit formation of embryonic boundaries such as separation of the cardinal embryonic germ layers, embryonic segmentation and fractal-like development of the branched tub- 
ular networks including blood vessels, lymphatics, and pulmonary architecture.

The erythropoietin-producing hepatocellular (Eph) family of receptor tyrosine kinases regulate a multitude of physicological and pathological processes. Noberini et al. [85] performed a high throughput screen of small molecules as potential ligands for the extracellular domain of the EphA4 receptor. These investigators found that a 2, 5-dimethyl-pyrrolyl benzoic acid derivative, as well as a number of other molecular ligands, could inhibit the interaction of EphA4 with a peptide ligand and with natural ephrin ligands. Among the investigational compounds, two isomers acted as competitive inhibitors selectively at the Eph-A4 and the closely related EphA2 receptor $[85 ; 86]$. These findings demonstrated that small inhibitory molecules could selectively target the Eph receptorephrin signaling system.

\section{PROSEPCTS FOR PROTECTIVE/MITIGATIVE THE- RAPIES: RESEARCH DEVELOPMENT NEEDS}

The core "forward-looking" question is as follows:

- Are the exogenous risk factors for gastroschisis modifiable?

Key corollary questions include

- How early can gastroschisis be diagnosed?

- Could early intervention allow mitigation that would reduce the severity of individual cases by the institution of some safe and at least partially effective therapy? In other words, could a therapy shift complex cases to be manifest as more straight-forward cases of gastroschisis?

- $\quad$ Ultimately in the future, could true preventive (risk reduction) interventions be proven and implemented to reduce some occurrences altogether?

While accelerated and delayed early embryonic growth in utero can be measured by ultrasound between 6 and ten gestational weeks [87], Khan et al. [88] summarized the current prospects for in utero gastroschisis imaging per se. Since gastroschisis represents a herniation of abdominal contents through a paramedian full-thickness abdominal fusion defect usually to the right side of the umbilical cord, then in early pregnancy, loops of bowel may be seen floating in the amniotic fluid. While gastroschisis is usually detected before 20 weeks of gestation by ultrasound, with transvaginal ultrasound, the diagnosis can be made as early as 12 weeks of gestation. Although antenatal ultrasound imaging is the primary means for diagnosis, detection rates are only about 70\%. Presumably, diagnostic performance is limited by both being operator dependent and due to artifacts in imaging such that visualization of a $2-5 \mathrm{~cm}$ defect in the right paraumbilical region to make the diagnosis of gastroschisis can be missed. Finally, while the anterior abdominal wall and umbilical cord insertion can be readily recognized on ante-natal scanning, the inner aspect of the anterior abdominal wall can be challenging to distinguish from the abdominal viscera.

If we focus on our two hypothesized molecular/cellular MOAs of Mechanosensitivity and Mechanotransduction
Signaling and Ephrin-Eph Receptor Signaling, then what are the plausible "druggable" molecules that might play a role in mediating the occurrence of gastroschisis, implying some role in development of skin, subcutaneous tissue, fascia, muscle, peritoneum, possibly smooth muscle and/or combinations of those tissues? Some unevaluated possibilities exist. One facile resource in such a search for druggable molecular targets is the Druggable Proteome section within the Human Protein Atlas [89; https:// proteinatlas.org/humanproteome/druggable].This resource is readily searchable for various functional and structural categories of human proteins. For the current purpose of seeking molecules that might be critical to normal or disordered development of the embryonic/fetal abdominal wall, we would pose queries to seek membrane-associated proteins that may relate to

a) linkage among adjacent cells or to extraellular constituents (Mechanosensitivity and Mechanotransduction Signaling) and

b) Ephrin-Eph Receptor Signaling molecules, and for which there are data indicating substantial expression in relevant tissues such as skeletal muscle or skin.

Using those simple cues, a modest number of druggable molecules can be nominated as candidates for further research and potential development as protective or mitigative therapeutics.

This database tool groups targets for FDA approved drugs by function as classes of enzymes, transporters, voltage-gated ion channels, G-protein coupled receptors, nuclear receptors, and CD markers. For the purpose of translational research in gastroschisis, cellular localization of targets may offer an important corollary clue as to potential relevance for this developmental disorder. If such structural and locational information heightens relevance, then integral membrane (IM; $n=250$ ), single pass transmembrane (SPTM; $n=101$ ) and IM/SPTM ( $n=9$ ) would be worthy of prioritizing for research attention. Among those groups (IM, SPTM and IM/SPTM), a few molecules appear to have some background data suggesting relevance to abdominal wall development/maldevelopment.

We do not claim that these nominees are known to mediate gastroschisis, nor would we exclude many other potential molecular pathways or key cellular mediators. Indeed, we hope to evoke new inquiries by investigators with a range of expertise and research skillsets to address the challenge and opportunity that these mechanistic prospects present.

As suggested by the prior section on Ephrin-Eph recepptor signaling, we suggest that this pathway may be involved in mediating abdominal wall development and could be modulated by medications or dietary component. In addition to reports $[85,86]$ about small molecule ligands for the EphA4 and EphA2 receptors, there are other candidates as small molecule Eph receptor ligands. The endogenous human compound lithocholic acid is a bile acid produced by gut bacterial flora. Giorgio et al. [90] have identified lithocholic acid (LCA) as a reversible comp- 
titive ligand that inhibits EphA2 receptor-ephrin-A1 binding and that LCA inhibited EphA2 phosphorylation induced by ephrinA1-Fc in several cell lines without affecting cell viability or other receptor tyrosine-kinase (EGFR,
VEGFR, IGFR1b, IRKb) activity. They noted that structurally related bile acids neither inhibited Eph-ephrin binding nor Eph phosphorylation.

Table 1. Potentially druggable membrane-associated human proteins in gastroschisis related to hypothesized MOAs of mechanosensitivity and mechanotransduction signaling or Ephrin-Eph receptor signaling

\begin{tabular}{|c|c|c|}
\hline Postulated MOA & $\begin{array}{l}\text { Nominated Druggable } \\
\text { Molecule(s) }\end{array}$ & Substantial expression in relevant tissue(s) \\
\hline \multicolumn{3}{|c|}{ Mechanosensitivity and } \\
\hline \multicolumn{3}{|c|}{ Mechanotransduction Signaling 1} \\
\hline & Integrin subunit alpha $\mathrm{V}$ & Skin, smooth muscle \\
\hline & Integrin subunit beta 1 & Smooth muscle \\
\hline & Integrin subunit beta 7 & Skeletal muscle, skin \\
\hline \multicolumn{3}{|c|}{ Ephrin-Eph Receptor Signaling² } \\
\hline & Ephrin A3 & Skin \\
\hline & Ephrin A4 & Skeletal muscle, skin \\
\hline & Ephrin A5 & Skeletal muscle, skin \\
\hline & Ephrin B1 & Skeletal muscle, skin \\
\hline & Ephrin B2 & Skeletal muscle, skin \\
\hline & Eph receptor A1 & Skeletal muscle, skin \\
\hline & Eph receptor A3 & Skeletal muscle \\
\hline & Eph receptor A4 & Skin \\
\hline & Eph receptor A7 & Skeletal muscle, skin \\
\hline & Eph receptor A10 & Skeletal muscle, skin \\
\hline & Eph receptor B2 & Skeletal muscle, skin \\
\hline & Eph receptor B3 & Skin \\
\hline & Eph receptor B4 & Skeletal muscle \\
\hline \multicolumn{3}{|c|}{ Other $(s)^{3}$} \\
\hline \multicolumn{3}{|c|}{ Matrix Metallopeptidases (MMPs) ${ }^{4}$} \\
\hline & MMP 14 & Skin \\
\hline & MMP 15 & Skeletal muscle, skin \\
\hline & MMP 16 & Skeletal muscle, skin \\
\hline
\end{tabular}

${ }^{1}$ Selected from 25 Integrin molecules.

${ }^{2}$ Selected from 22 Ephrin and Eph receptor molecules.

${ }^{3}$ Various other groups of molecules could be considered; MMPs are shown as an illustrative group.

${ }^{4}$ Selected from 23 MMPs.

A final speculative suggestion where one or more small molecules might mediate protection against gastroschisis occurrence is a recent report about gastroschisis and maternal intake of phytoestrogens [91]. In that report, Wadhwa et al. evaluated whether the risk of gastroschisis was associated with maternal dietary intake of phytoestrogens. In the National Birth Defects Prevention Stud$\mathrm{y}$, these investigators analyzed data on mothers of 409 gastroschisis cases and 3,007 controls with births in 2005-2010. From validated maternal food frequency questionnaire data, logistic regression analyses for each phytoestrogen was adjusted for maternal energy intake, age, BMI, race/ethnicity, and first-trimester smoking. This analysis showed that biochanin A, formononetin, and coumestrol had significant non-linear associations with gastroschisis. For these compounds, lower intakes were associated with increased risk and associations were not significant for the other phytoestrogens.

Since this profile of potentially protective isoflavones and coumestans is somewhat characteristic of those that occur in the group of legumes that include red clover, we suggest that this group of plants would merit systematic phytopharmacognosy research into prevention of gas- troschisis in animal models and ultimately in human preventive clinical trials.

\section{SUMMARY}

In order to reduce the future occurrence and /or severity of cases of gastroschisis, what public health, lifestyle, clinical care, and therapeutic research and development options appear to be plausible? We offer the following recommendations as worthy of consideration knowing that many of these will surely not be applicable in all circumstances or for all gravidas:

1) Young maternal age - Provide younger adult women and their male partners with information regarding various advantages (including lowered risk of gastroschisis) for delaying childbearing along with access to contraception choices.

2) Pre-pregnancy and gestational nutrition - Adopt interventions to attain better nutritional status regarding both diet quality (intake of fruits and vegetables) and adequacy of caloric intake particularly for younger gravidas.

3) Recasting "prenatal care" to routinely include acessible preconceptional counseling, leading into early access to prenatal care per se could 
a. reduce embryonic exposures to commonly use$\mathrm{d}$ medications such as aspirin, oral contraceptives, ibuprofen, decongestants, venlafaxine, antiherpetic medications (acyclovir, valacyclovir or famciclovir), antidepressants, topical antivirals;

b. reduce embryonic exposures to occupational/ residential chemicals (polycyclic aromatic hydrocarbons, solvents, colorants, various agricultural chemicals);

c. guidance to avoid episodes of excessive heat stress;

d. possibly detection and reduction of episodes of domestic violence;

e. optimize pre-conception control of diabetes as well as early detection and control of gestational diabetes;

f. early detection and treatment of chlamydia trachomatis;

g. Very early ultrasound assessment of fetal anatomy is the most plausible means for earliest detection of a gastroschisis defect, which would be a requisite for instituting treatment with any potential or (hopefully) proven therapy that may mitigate the severity of gastroschisis cases.

4) Lifestyle/recreational drugs - Use all valid means to minimize prepregnancy and gestational use of tobacco, alcohol, cannabis and "recreational" drugs (cocaine, methamphetamine, etc.) of abuse.

5) Broadly implore research colleagues to

a. take on the challenge of defining the underlying cellular and molecular mechanisms by which various environmental factors impact the risk and severity of gastroschisis and

b. to drive those insights across translational boundaries to provide one or more safe and efficacious preventive or mitigative therapeutics for this developmental disease.

While we have hypothesized that druggable molecules in the Mechanosensitivity and Mechanotransduction Signaling and Ephrin-Eph Receptor Signaling pathways should be highly attractive unique research and development opportunities, the diverse range of exogenous factors implicated in the occurrence of gastroschisis will likely mean that multiple signaling pathways may be relevant in various distinct subsets of causal exposures.

\section{CONFLICT OF INTERESTS}

The authors declare that there is no conflict of interest regarding the publication of this paper.

\section{REFERENCES}

1. Parker SE et al. for the National Birth Defects Prevention Network. Updated national birth prevalence estimates for selected birth defects in the United States, 2004-2006. Birth Defects Res A Clin Mol Teratol. 2010; 88(12):1008-16.
2. Bugge M, Holm NV. Abdominal wall defects in Denmark, 1970-89. Paediatr Perinat Epidemiol. 2002:16 (1 ):73-81.

3. Jones AM et al. Increasing prevalence of gastroschisis 14 States, 1995-2012. MMWR. January 22, 2016: 65(2):23-6.

4. Collins SR et al. The rising prevalence of gastroschisis and omphalocele in Tennessee. J Pediatr Surg. 2007 ; 42:1221-4.

5. Bugge $\mathrm{M}$ et al. Abdominal wall defects in Greenland 1989-2015. Birth Defects Res. 2017; 109(11):836-42. Doi:10.1002/bdr2. 1025.

6. Lane $\mathrm{C}$ et al. A population-based study of prevalence trends and geospatial analysis of hypospadias and cryptorchidism compared with non-endocrine mediated congenital anomalies. J Pediatr Urol. 2017; 13(3) :284.e1-7. doi: 10.1016/j.jpurol.2017.02.007.

7. Mastroiacovo $\mathrm{P}$ et al. Gastroschisis and associated defects: an international study. Am J Med Genet Part A. $2007 ; 143 \mathrm{~A}: 660-71$

8. Keys $\mathrm{C}$ et al. Gastroschisis: the cost of an epidemic. J Pediatr Surg. 2008; 43:654-7.

9. de Buys Roessingh AS et al. Predictive factors at birth of the severity of gastroschisis. World J Gastrointest Pathophysiol 2015; 6(4):228-34. Available from: URL :http://www.wjgnet.com/2150-5330/full/v6/i4/ 228.htm.DOI:http://dx.doi.org/10.4291/wjgp .v6.i4.228

10. Kassa A-M, Lilja HE. Predictors of postnatal outcome in neonates with gastroschisis. J Pediatr Surg. 2011; 46:2108-14.

11. Lund $\mathrm{CH}$ et al. Gastroschisis: incidence, complications, and clinical management in the neonatal intensive care unit. J Perinat Neonatal Nurs. 2007; 21 (1): 63-8.

12. Bergholz $\mathrm{R}$ et al. Complex gastroschisis is a different entity to simple gastroschisis affecting morbidity and mortality-A systematic review and meta-analysis. J Pediatr Surg. 2014; 49:1527-32.

13. Youssef $\mathrm{F}$ et al. Flap versus fascial closure for gastroschisis: a systematic review and meta-analysis. J Pediatr Surg. 2016; 51(5):718-25. Doi:10.1016/j.jpedsurg.2016.02.010.

14. Opitz JM Invited Comment: Gastroschisis. Am J Med Genet Part A. 2007; 143A:635-8.

15. Drongowski RA et al. Contribution of demographic and environmental factors to the etiology of gastroschisis: a hypothesis. Fetal Diagn Ther. 1991; 6(12):14-27.

16. Payne NR et al. Growth restriction in gastroschisis: quantification of its severity and exploration of a placental cause. BMC Pediatrics 2011, 11:90 http: //www.biomedcentral.Com/1471-2431/11/ 90.

17. Mac Bird T et al. Demographic and environmental risk factors for gastroschisis and omphalocele in the National Birth Defects Prevention Study. J Pediatr Surg. 2009; 44(8):1546-51. doi: 10.1016/j.jpedsurg. 2008. 10.109.

18. Duong HT et al. Maternal use of hot tub and major structural birth defects. Birth Defects Res A Clin Mol Teratol. 2011; 91(9):836-41.doi:10.1002/bdra. 20831.

19. Agopian AJ et al. A case-control study of maternal bathing habits and risk for birth defects in offspring. Environ Health. 2013; 12: 88. doi:10.1186/1476-069X12-88. 
20. Lin S et al. Maternal occupation and the risk of major birth defects: a follow-up analysis from the National Birth Defects Prevention Study. Int J Hyg Environ Health. 2013; 216(3):317-23. doi: 10.1016/j.ijheh. 2012.05.006.

21. Ortega-García JA et al. Violence against women and gastroschisis: a case-control study. Int J Environ Res Public Health. 2013; 10(10):5178-90.doi:10.3390/ ijerph 10105178.

22. Skarsgard ED et al. Maternal risk factors for gastroschisis in Canada. Birth Defects Res A Clin Mol Teratol. 2015; 103(2):111-8. doi:10.1002/ bdra. 23349.

23. Bugge $\mathrm{M}$ et al. Monozygotic twins discordant for gastroschisis: case report and review of the literature of twins and familial occurrence of gastroschisis. Am J Med Genet. 1994; 52(2): 223-6.

24. Hillebrandt $S$ et al. Genetic analysis of the cause of gastroschisis in the HLG mouse strain. Mutat Res. 1996 Nov $11 ; 372(1): 43-51$.

25. Streffer C. Transgenerational transmission of radiation damage: genomic instability and congenital malformation. J Radiat Res. 2006; 47 Suppl B: B19-24.

26. Feldkamp ML et al. AEBP1 gene variants in infants with gastroschisis. Birth Defects Res A Clin Mol Teratol. 2012; 94(9):738-42. doi:10. 1002/bdra. 23041.

27. Padula AM et al. Gene variants as risk factors for gastroschisis. Am J Med Genet A. 2016; 170 (11): 27882802. doi: 10.1002/ajmg.a. 37883.

28. Makhmudi A et al. Effects of MTHFR c.677C>T, F2 c.20210G >A and F5 Leiden polymorphisms in gastroschisis. J Invest Surg. 2016; 29(2):88-92. doi:10. 3109 /08941939. 2015.1077908.

29. Rittler $\mathrm{M}$ et al. Gastroschisis is a defect of the umbilical ring: evidence from morphological evaluation of stillborn fetuses. Birth Defects Research (Part A). 2013; 97:198-209.

30. Bargy F, Beaudion S. Comprehensive developpmental mechanisms in gastroschisis. Fetal Diagn Ther 2014;36:223-230 DOI: 10.1159 /000360080

31. Goldbaum $\mathrm{G}$ et al. Risk factors for gastroschisis. Teratology. 1990 Oct; 42(4):397-403.

32. Chabra S, Hall BD. A cluster study of gastroschisis: single center experience. J Ky Med Assoc. 2008 Aug; 106(8):361-5.

33. Rasmussen SA, Frías JL. Non-genetic risk factors for gastroschisis. Am J Med Genet C Semin Med Genet. 2008; 148C (3):199-212. doi:10. 1002/ajmg.c.30175.

34. Lammer EJ et al. Gastroschisis: a gene-environment model involving the VEGF-NOS3 pathway. Am J Med Genet C Semin Med Genet. 2008; 148C (3):213-8.doi: 10.1002/ajmg. c. 30182.

35. Werler MM et al. Demographic, reproductive, medical, and environmental factors in relation to gastroschisis. Teratology. 1992; 45 (4):353-60.

36. Torfs CP et al. Maternal medications and environmental exposures as risk factors for gastroschisis. Teratology. 1996; 54(2):84-92.

37. Hume RF et al. Vascular disruption birth defects and history of prenatal cocaine exposure: a case control study. Fetal Diagn Ther. 1997; 12(5):292-5.

38. Morrison JJ et al. Recreational drugs and fetal gastroschisis: maternal hair analysis in the periconceptional period and during pregnancy. BJOG. 2005; 112 (8):1022-5.

39. David AL et al. A case-control study of maternal periconceptional and pregnancy recreational drug use and fetal malformation using hair analysis. PLoS One. 2014 Oct 31; 9(10): e111038. doi: 10.1371/journal. pone. 0111038.

40. Short T et al. Gastroschisis trends and ecologic link to opioid prescription rates-United States, 2006-2015 .MMWR. 2019; 68:31-36.

41. Burdan $\mathrm{F}$ et al. Celosomy is associated with prenatal exposure to cyclooxygenase inhibitors. Pharmacol Res. 2006; 53(3):287-92.

42. Elliott $\mathrm{L}$ et al. Case-control study of a gastroschisis cluster in Nevada. Arch Pediatr Adolesc Med. 2009; 163 (11):1000-6. doi: 10.1001/archpediatrics. 2009. 186.

43. Richardson $\mathrm{S}$ et al. Associations between periconceptional alcohol consumption and craniosynostosis, omphalocele, and gastroschisis. Birth Defects Res A Clin Mol Teratol. 2011; 91(7):623-30.doi: 10.1002/ bdra 20823.

44. Hackshaw A et al. Maternal smoking in pregnancy and birth defects: a systematic review based on 173 687 malformed cases and 11.7 million controls. Hum Reprod Update.2011; 17(5):589-604.doi: 10.1093/ humupd/dmr022.

45. Jenkins MM et al. Maternal smoking, xenobiotic metabolizing enzyme gene variants, and gastroschisis risk. Am J Med Genet A. 2014; 164A (6):1454-63. doi: 10.1002/ajmg.a. 36478.

46. van Gelder MM et al. Using Bayesian models to assess the effects of under-reporting of cannabis use on the association with birth defects, national birth defects prevention study, 1997-2005. Paediatr Perinat Epidemiol. 2014; 28 (5):424-33. doi: 10.1111/ppe. 12140.

47. Polen KN et al. Association between reported venlafaxine use in early pregnancy and birth defects, national birth defects prevention study, 1997-2007. Birth Defects Res A Clin Mol Teratol. 2013; 97(1):2835. doi: 10.1002/bdra. 23096.

48. Ahrens KA et al. Antiherpetic medication use and the risk of gastroschisis: findings from the National Birth Defects Prevention Study, 1997-2007. Paediatr Perinat Epidemiol. 2013; 27 (4):340-5. doi: 10.1111/ppe. 12064.

49. Given JE et al. EUROmediCAT signal detection: an evaluation of selected congenital anomaly-medication associations. Br J Clin Pharmacol. 2016; 82(4): 1094109. doi: 10.1111 /bcp. 12947.

50. Given JE et al. Gastroschisis in Europe - A case-malformed-control study of medication and maternal illness during pregnancy as risk factors. Paediatr Perinat Epidemiol. 2017; 31 (6):549-559. doi:10.1111/ppe. 12401.

51. Feldkamp ML et al. Chlamydia trachomatis is responsible for lipid vacuolation in the amniotic epithelium of fetal gastroschisis. Birth Defects Res. 2017; 109 (13):1003-1010. doi: 10.1002/bdr2.1062.

52. Singh J. Gastroschisis is caused by the combination of carbon monoxide and protein-zinc deficiencies in mice. Birth Defects Res B Dev Reprod Toxicol. 2003; 68 (4):355-62.

53. Torfs CP et al. Selected gene polymorphisms and their interaction with maternal smoking, as risk factors for gastroschisis. Birth Defects Res A Clin Mol Teratol. 2006 Oct; 76(10):723-30.

54. Waller DK et al. Prepregnancy obesity as a risk factor for structural birth defects. Arch Pediatr Adolesc Med. 2007; 161(8):745-750. 
55. Siega-Riz AM et al. The joint effects of maternal prepregnancy body mass index and age on the risk of gastroschisis. Paediatric and Perinatal Epidemiology 2009; 23: 51-57.

56. Paranjothy $S$ et al. The role of maternal nutrition in the etiology of gastroschisis: an incident case-control study. Inter J Epidemiol 2012; 41:1141-1152 doi: 10.1093/ije/dys092.

57. Dolk $\mathrm{H}$ et al. Risk of congenital anomalies near hazardous-waste landfill sites in Europe: the EUROHAZCON study. Lancet. 1998 Aug 8; 352(9126): 423-7.

58. Fielder HM et al. Assessment of impact on the health of residents living near the Nanty-Gwyddon landfill site: a retrospective analysis. BMJ. 2000 Jan 1; 320 (7226):19-22.

59. Elliott P et al. Risk of adverse birth outcomes in populations living near landfill sites. BMJ. 2001 Aug 18; 323(7309):363-8.

60. Root ED, Emch ME. Tracing drinking water to its source: An ecological study of the relationship between textile mills and gastroschisis in North Carolina. Health Place. 2010; 16(5):794-802. doi: 0.1016/j. health-place.2010.04.004.

61. Lupo PJ et al. Maternal occupational exposure to polycyclic aromatic hydrocarbons: effects on gastroschisis among offspring in the National Birth Defects Prevention Study. Environ Health Perspect.2012;120(6): 910-5.doi:10.1289/ehp.1104305.

62. Wangikar PB et al. Effect in rats of simultaneous prenatal exposure to ochratoxin A and aflatoxin B1. I. maternal toxicity and fetal malformations. Birth Defects Res B Dev Reprod Toxicol. 2004; 71(6):343-51.

63. Van Dorp DR et al. Teratogens inducing congenital abdominal wall defects in animal models. Pediatr Surg Int. 2010; 26(2):127-39. doi: 10.1007/s00383-009 -2482-z.

64. Waller SA et al. Agricultural-related chemical exposures, the season of conception, and risk of gastroschisis in Washington State. Am J Obstet Gynecol 2010; 202:41.e1-6.

65. Agopian AJ et al. Maternal residential atrazine exposure and gastroschisis by maternal age. Matern Child Health J. 2013;17(10):1768-75.doi:10.1007/s10995012-1196-3.

66. Joshi N et al. Developmental abnormalities in chicken embryos exposed to N-nitrosoatrazine. J Toxicol Environ Health A. 2013; 76 (17): 1015-22.doi:10.1080/ 15287394.2013. 831721.

67. Kielb C et al. Maternal periconceptional occupational exposure to pesticides and selected musculoskeletal birth defects. Int J Hyg Environ Health. 2014; 217(23):248-54.doi:10.1016/j.ijheh. 2013.06. 003

68. Shaw GM et al. Early pregnancy agricultural pesticide exposures and risk of gastroschisis among offspring in the San Joaquin Valley of California. Birth Defects Res A Clin Mol Teratol. 2014; 100(9):686-94.doi: 10. 1002/bdra. 23263.

69. Feldkamp M et al. Development of Gastroschisis: Review of Hypotheses, a novel hypothesis, and implications for Research. Am J Med Genet Part A. 2007; 143 A: 639-652.

70. Chambers $\mathrm{C}$ et al. Novel risk factor in gastroschisis: change of paternity. Am J Med Genet Part A. 2007; 143:53-659.

71. Bateman ME et al. The effects of endocrine disruptors on adipogenesis and osteogenesis in mesenchymal stem cells: a review. Front. Endocrinol. 2016; 7: 171. doi: 10.3389 / fendo. 2016.00171.
72. Ladoux B, Mège R. Mechanobiology of collective cell behaviours. Nat Rev Mol Cell Biol 2017; 18:743-757.

73. Tatapudy $\mathrm{S}$ et al. Cell fate decisions: emerging roles for metabolic signals and cell morphology. EMBO reports. 2017; 18 (12): 2105-2117.

74. Uhler C, Shivashankar G. Regulation of genome organization and gene expression by nuclear mechanotransduction. Nat Rev Mol Cell Biol. 2017; 18:717727.doi: 10.1038/nrm.2017.101.

75. Vining K, Mooney D. Mechanical forces direct stem cell behavior in development and regeneration. Nat Rev Mol Cell Biol. 2017; 18:728-742.

76. Mongera A et al. A fluid-to-solid jamming transition underlies vertebrate body axis elongateion. Nature. 2018;https://doi.org/10. 1038 /s41586-018-0479-2 (2018).

77. Lenne P-F, Trivedi V. Melting sculpts the embryo's body.Nature.2018;https://doi.org/10.1038/ d41586 -018-06108-7163.

78. Sawyer JK et al. A contractile actomyosin network linked to adherens junctions by Canoe/afadin helps drive convergent extension. Mol. Biol. Cell 22, 24912508 (2011).

79. Sawyer JK et al. The Drosophila afadin homologue Canoe regulates linkage of the actin cytoskeleton to adherens junctions during apical constriction. J. Cell Biol. 186, 57-73 (2009).

80. Mandai K. et al. Afadin: a novel actin filament-binding protein with one PDZ domain localized at cadherin-based cell-to-cell adherens junction. J. Cell Biol. 139, 517-528 (1997).

81. Ikeda $\mathrm{W}$ et al. Afadin: a key molecule essential for structural organization of cell-cell junctions of polarized epithelia during embryogenesis. J. Cell Biol. 146, 1117-1132 (1999).

82. Zhadanov $\mathrm{AB}$ et al. Absence of the tight junctional protein AF-6 disrupts epithelial cell-cell junctions and cell polarity during mouse development. Curr. Biol. 9, 880-888 (1999).

83. Choi W. et al. Remodeling the zonula adherens in response to tension and the role of afadin in this response. J. Cell Biol. 213, 243-260 (2016).

84. Kania A, Klein R. Mechanisms of ephrin-Eph signalling in development, physiology and disease Nat Rev Mol Cell Biol. 2016; 17:240-256.

85. Noberini R et al. Small molecules can selectively inhibit Ephrin binding to the EphA4 and EphA2 receptors. J Biol Chem. 2008; 283(43): 29461-29472.

86. Qin $\mathrm{H}$ et al. Crystal structure and NMR binding reveal that two small molecule antagonists target the high affinity Ephrin-binding channel of the EphA4 receptor. J Biol Chem. 2008; 283 (43); 29473-29484.

87. Parisi F et al. Early first trimester maternal 'high fish and olive oil and low meat' dietary pattern is associated with accelerated human embryonic developpment. Eur J Clin Nutr. 2018; 72:1955-62.

88. Khan A et al. Gastroschisis imaging. Medscape. 2016; 1-9.https://emedicine.medscape.com/article /403800-print.

89. The Human Protein Atlas; The druggable proteome. https://www.proteinatlas.org/humanproteome/dru ggable

90. Giorgio $\mathrm{C}$ et al. Lithocholic acid is an Eph-ephrin ligand interfering with Eph-kinase activation. PLoS One 2011; 6(3): e18128. doi:10. 1371/journal. pone. 0018128 
91. Wadhwa EL et al. Gastroschisis and maternal intake of phytoestrogens. Am J Med Genet A. 2016; 170(8): 2078-82.doi:10.1002/ajmg.a. 37659.

How to cite this article:

Hughes $\mathrm{C}$ and Adibe $\mathrm{O}$. The increasing prevalence of gastroschisis: associated factors, possible mechanisms, and potential mitigative interventions. Glob Clin Transl Res. 2019; 1(1):4-20

Copyright (C) 2019 by the Global Clinical and Translational Research 